\title{
ARTICLE \\ Adolescent alcohol exposure produces sex differences in negative affect-like behavior and group I mGluR BNST plasticity
}

\author{
C. R. Kasten ${ }^{1}$, K. L. Carzoli ${ }^{1}$, N. M. Sharfman ${ }^{1}$, T. Henderson ${ }^{1}$, E. B. Holmgren ${ }^{1}$, M. R. Lerner ${ }^{1}$, M. C. Miller ${ }^{1}$ and T. A. Wills ${ }^{1,2}$
}

\begin{abstract}
Adolescent alcohol exposure increases the risk of developing alcohol use disorders (AUDs), yet the mechanisms responsible for this vulnerability remain largely unknown. One potential target for alcohol-induced changes is the circuitry that modulates negative affect and stress, two sexually dependent drivers of alcohol relapse. The bed nucleus of the stria terminalis (BNST) is a sexually dimorphic region that critically regulates negative affective- and stress-induced relapse. Group I metabotropic glutamate receptors (mGluR) are a target of interest due to their regulation of stress, anxiety behaviors, and BNST plasticity. The current studies investigate sex-dependent sensitivity to the effects of adolescent intermittent ethanol vapor exposure (AIE) on negative affect during acute and protracted alcohol withdrawal and following stress in adulthood. This work also assessed whether BNST group I mGluR-mediated long-term depression (LTD) was disrupted at these timepoints. During acute withdrawal, AIE altered LTD induced by the group I mGluR antagonist DHPG in females, but not males. During adulthood, stress unmasked persistent changes in DHPGinduced LTD and behavior that were not present under basal conditions. Females with an AIE history demonstrated enhanced negative affective-like behavior in the novelty-induced hypophagia test following restraint stress-a phenotype that could be blocked with systemic mGluR5 allosteric antagonism via MTEP. Conversely, males with an AIE history demonstrated elevated freezing in a contextual fear conditioning paradigm. These studies demonstrate long-lasting, sex-dependent phenotypes produced by AIE and suggest pharmaceutical interventions for alcohol use and comorbid disorders may be more effective if designed with sex differences in mind.
\end{abstract}

Neuropsychopharmacology (2020) 45:1306-1315; https://doi.org/10.1038/s41386-020-0670-7

\section{INTRODUCTION}

Growing evidence supports the presence of sex and gender differences in various aspects of alcohol use and abuse. Physiologically, sex disparities exist in how the body processes alcohol [1, 2], with men demonstrating greater physical withdrawal symptoms. However, women exhibit heightened propensity to use alcohol as a regulator of negative affective disorders [3-5], lending support to the increased rate of comorbid alcohol use disorders (AUDs) and anxiety/depressive disorders among women $[6,7]$.

Affective disorders tend to emerge during adolescence-a developmental period that also marks the initiation of alcohol use. Drinking during this stage is one of the biggest predictors for later development of an alcohol use disorder [8,9]. Negative affect and stress are primary drivers of alcohol relapse [10]. Thus, adolescent alcohol exposure may produce persistent neurophysiological alterations that enhance susceptibility to negative affective states and stress circuitry [11]. The current work sought to determine the effects of adolescent intermittent ethanol vapor (AIE) on acute and protracted negative affect phenotypes induced by alcohol withdrawal and stress. Negative affect indicates core vulnerabilities that underlie common dimensions of highly comorbid emotional disorders, including depression, anxiety, and enhanced threat monitoring $[12,13]$. Analogous preclinical models of these tasks include the elevated plus maze, open field activity, the noveltyinduced hypophagia task, and contextual fear learning [14-16].

In part, these emotional behaviors are regulated by the bed nucleus of the stria terminalis (BNST). The BNST is a highly sexually dimorphic brain region that is critically involved in negative affectand stress-induced relapse to alcohol $[17,18]$. Thus, alcoholinduced changes in BNST plasticity could provide the molecular mechanism of sex differences in negative affective phenotypes that develop over adolescence. Our previous work indicated that drugs and alcohol modulate BNST plasticity and excitatory transmission via $\mathrm{N}$-methyl-D-aspartate receptors (NMDARs) in adult males $[19,20]$. More recently, we demonstrated that acute and protracted AIE withdrawal enhances NMDAR-mediated plasticity in males, but not in females, even though both sexes have enhanced sEPSC frequency [21]. We therefore hypothesize that an alternate glutamatergic signaling mechanism, like group I metabotropic glutamate receptors (mGluR1 and mGluR5), could mediate AIE-induced neuroadaptation in females. Group I mGluRs regulate BNST plasticity $[22,23]$, have been implicated in numerous alcohol-related behaviors $[24,25]$, and mediate

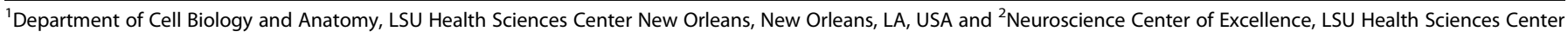
New Orleans, New Orleans, LA, USA

Correspondence: T. A. Wills (twills@lsuhsc.edu)

These authors contributed equally: C. R. Kasten, K. L. Carzoli

Received: 28 October 2019 Revised: 27 March 2020 Accepted: 31 March 2020

Published online: 8 April 2020 
symptoms of negative affect [26]. Moreover, mGluR-dependent long term depression (mGluR-LTD) in the hippocampus and BNST of adult male mice is blunted by alcohol withdrawal and cocaine exposure, respectively $[22,27]$.

The current study evaluated the influence of AIE on negative affective-like phenotypes and group I mGluR signaling using a combined behavioral, pharmacological, and electrophysiological approach. We also evaluated the persistence of behavioral and physiological adaptations under basal conditions and following an acute stress challenge in adulthood-a cue known to precipitate alcohol relapse. We used male and female mice because sex differences across these affective behavioral tasks following protracted alcohol withdrawal have not been widely reported $[11,26]$. Elucidating the mechanisms of sex-specific changes in the BNST following alcohol and stress, as well as associated behavioral phenotypes, may lead to more effective approaches for AUD treatment.

\section{MATERIALS AND METHODS}

Animals

Three-week-old [postnatal day (P)21] C57BL/6J mice were obtained from Jackson Laboratories (Bar Harbor, ME). Males and females were housed separately, in groups of $4-5$, with food and water available ad libitum. All procedures were approved by the Animal Care and Use Committee at Louisiana State University Health Sciences Center.

\section{AIE vapor exposure}

Mice were exposed to AIE as previously described [21]. Briefly, ethanol chamber exposure occurred from 1600 to 0800 the following day, which allowed for the reliable obtainment of blood ethanol concentrations in the range of $150-185 \mathrm{mg} / \mathrm{dL}$. AIE protocol was run for two, $4 \mathrm{~d}$ cycles of $16 \mathrm{~h}$ in-chamber sessions and $8 \mathrm{~h}$ outof-chamber sessions separated by $3 \mathrm{~d}$ off from P30 to 41). Male and female mice were exposed in separate vapor chambers.

Elevated plus maze

EPM was performed $4-5 \mathrm{~h}$ following the final AIE vapor chamber session. The EPM test was run as previously described [28], see Supplemental materials for details.

Slice preparation

Brain slices for all electrophysiological and western blot experiments were collected during acute withdrawal (4-5 h following removal from the vapor chambers) or in adulthood (P70-75). Mice were transported from the animal colony to the laboratory and placed in a sound-attenuated cubicle for $1 \mathrm{~h}$. Coronal brain slices $(300 \mu \mathrm{m}$ thickness for electrophysiology and $500 \mu \mathrm{m}$ thickness for western blot analysis) containing the dorsolateral BNST (dIBNST; bregma, $0.26-0.02 \mathrm{~mm}$ ) were obtained using a vibrating microslicer (Leica Biosystems, Bannockburn, IL) as previously described [21].

Field potential recordings

After sectioning, slices were transferred to a heated holding chamber $\left(\sim 29^{\circ} \mathrm{C}\right)$ containing oxygenated artificial cerebral spinal fluid for $1 \mathrm{~h}$ prior to field recordings. Slice transfer, LTD induction, field excitatory postsynaptic potentials (fEPSPs) recordings, and data handling has been previously described [20]. After acquisition of a stable baseline, dihydroxyphenylglycine (DHPG; group I mGluR agonist, $100 \mu \mathrm{M}, 5 \mathrm{~min}$ ), methoxamine (a1-AR agonist, $100 \mu \mathrm{M}, 15 \mathrm{~min})$, or (2R)-amino-5-phosphonovaleric acid; (2R)amino-5-phosphonopentanoate (AP5; NMDAR antagonist, $50 \mu \mathrm{M}$, 20-40 min) was applied.

Western blotting

For protein extraction, $0.33 \mathrm{~mm}$ tissue punches were obtained from the dIBNST $4-5 \mathrm{~h}$ following the final vapor chamber session.
Samples were homogenized and Western blot analysis was performed as previously described [21]. To combine blocks of experiments across blots, signals were normalized to GAPDH (1:10,000; Abcam, Cambridge, MA) and calculated as a percentage of the control values on their respective blot. The following primary antibodies were used: GluA2 (1:2000; Millipore Sigma, Burlington, MA) and mGluR5 (1:3000; Millipore Sigma).

Open field activity

In adult mice (P70+), open field activity in the contextual fear apparatus was quantified for 3 min prior to shock administration to assess basal anxiety-like activity. Anxiety-like activity was quantified as the \% of total distance traveled in the center of the chamber.

\section{Novelty-induced hypophagia}

The NIH task was run between P70-85. Procedures were modified from a previously published protocol [29], see Supplemental materials for details. On the test day, mice were exposed to drug [no injection, vehicle, or $30 \mathrm{mg} / \mathrm{kg}$ MTEP (mGluR5 negative allosteric modulator, i.p., Tocris)] $30 \mathrm{~min}$ prior to restraint stress (see below). No injection + stress mice were run through the EPM at P45.

\section{Contextual fear conditioning}

Fear conditioning was conducted in a separate cohort of animals at $\mathrm{P} 70+$. Procedures followed a previously published protocol [30] using low-intensity foot shocks to detect any enhanced threat monitoring behavior (see Supplemental materials for details). Fear acquisition was quantified as the difference in freezing between pre- and post-shock. Fear extinction was quantified as the difference in freezing between post-shock during acquisition and during $\min 1,8$, and 15 of the extinction session (no shock presented).

Acute restraint stress

Adult mice were restrained in well-ventilated $50-\mathrm{mL}$ conical tubes and left undisturbed for $1 \mathrm{~h}$. Control mice remained in the home cage. After the restraint period, mice were transferred to their cages for $1 \mathrm{~h}$ before behavioral tests or slicing for electrophysiology.

Statistical analysis

Mice excluded from behavioral analyses are detailed in Supplementary Table 1 . All values are presented as means \pm SEM with $p<0.05$ considered significant, adjusted for post hoc analyses. Normality and the equality of variances were assessed, and statistical tests (unpaired $t$ tests and one- or two-way ANOVAs) were chosen accordingly. Several a priori hypotheses that $\mathrm{NIH}$ effects would be stress-dependent were made based on previous electrophysiology data [21]. Alpha-adjusted Sidak tests comparing air- vs. AlE-history within stress status were run to test these hypotheses.

\section{RESULTS}

AIE withdrawal promotes an anxiety-like phenotype in the elevated plus maze

Since heightened anxiety is a hallmark of alcohol withdrawal in humans and animal models [31], we tested activity on the EPM during acute AIE withdrawal (Fig. 1a). AIE withdrawal promoted a robust anxiety-like phenotype in both sexes, significantly reducing \% time spent in the open arms [females: $t(29)=2.15$, $p<0.05$, Fig. 1b; males: $t(17)=2.90, p<0.01$, Fig. $1 \mathrm{~d}$ ]. The total number of arm entries was also reduced by AIE, indicating AIE withdrawal induced hypo-locomotion in both sexes [females: $t(29)=5.08, p<0.001$, Fig. 1c; males: $t(17)=3.45, p<0.01$, Fig. 1e]. 
A)

AIE: P30-41

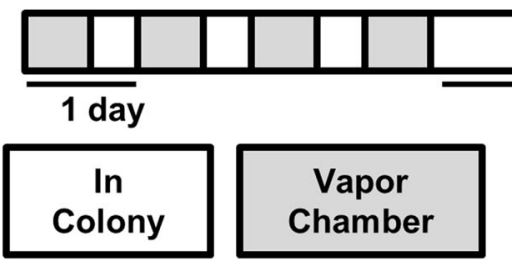

3 days

\section{EPM 5hrs after removal from vapor chamber}

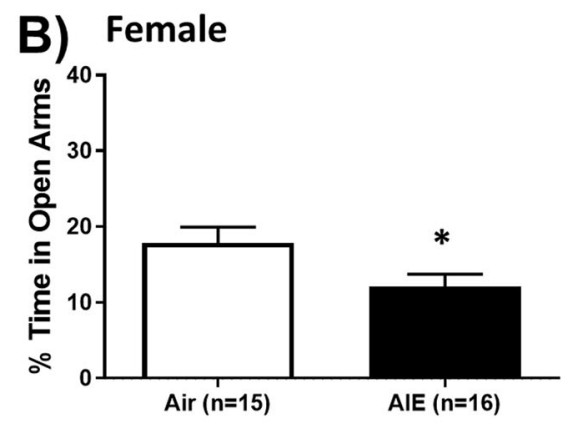

\section{D) Male}

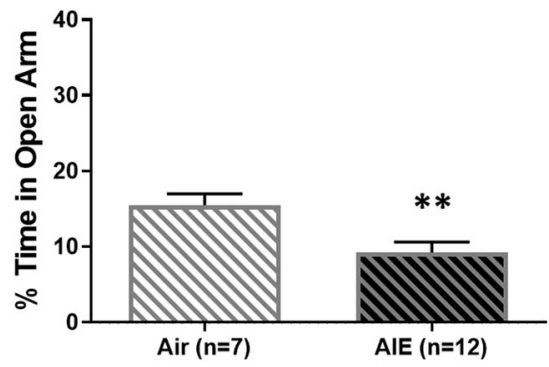

C) Female

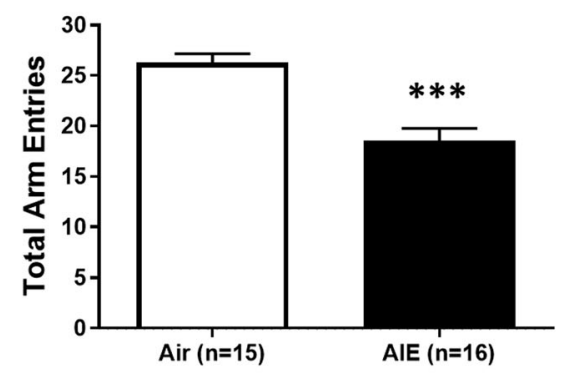

E) Male

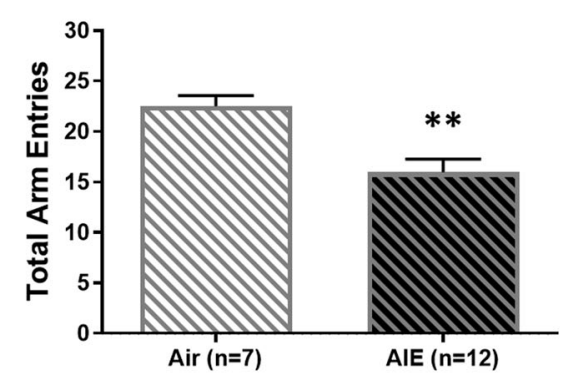

Fig. 1 Acute withdrawal from AIE increases anxiety-like activity on the EPM in both sexes. a Schedule of ethanol and water vapor exposure. Mice were exposed to volatilized water (air) or ethanol (AIE) for $16 \mathrm{~h} /$ day for two, 4-d blocks. The percentage of total time spent in the open arms was reduced during AIE withdrawal in female (b) and male (d) mice, as was the total number of arm entries (c, e, respectively). Data are shown as mean \pm SEM. Significantly different than the respective air group at ${ }^{*} p<0.05,{ }^{* *} p<0.01,{ }^{* * *} p<0.001$.

AIE withdrawal impairs group I mGluR-mediated depression in the dIBNST of female mice

Alcohol-induced changes in glutamatergic plasticity in the BNST are a known contributor to negative affect [32]. AlE-induced alterations in NMDAR-mediated BNST plasticity are present in males, but not females, suggesting an alternative glutamatergic target in females [21]. Therefore, the next set of experiments examined group I mGluR-dependent LTD in the dIBNST using DHPG (DHPG-LTD, group I mGluR agonist, 5 min, $100 \mu \mathrm{M}$ ) during AIE withdrawal (Fig. 2a). In females, we observed a significant difference in DHPG-LTD between adolescent treatment groups $[F(1,14)=32.37, p<0.0001]$ and LTD timepoint [early or late phase of LTD, $F(1,14)=58.15, p<0.0001$ ] with no interaction $(p>0.05$, Fig. 2b). At both timepoints, DHPG-LTD was significantly reduced in AIE-treated females compared to air controls (early: Sidak $p<$ 0.01 , late: $p<0.01)$. In contrast, there was no effect of adolescent treatment in males ( $p>0.05$, Fig. $2 \mathrm{e})$. These results suggest that AIE selectively impairs induction and maintenance of DHPG-LTD in the dIBNST of female, but not male, mice.

AIE withdrawal reduces GluA2 expression in the dIBNST of female mice and alters AMPAR-mediated fEPSP amplitude

BNST DHPG-LTD is dependent on the activation of mGluR5 and internalization of AMPARs in most brain regions [22]. To see if these molecular correlates of DHPG-LTD were disrupted by AIE, western blot analysis was performed to measure mGluR5 and GluA2 (AMPAR subunit) expression in the dIBNST during AIE withdrawal (Fig. 2a; representative blots shown in Supplementary Fig. 1). In females, AIE had no effect on mGluR5 expression (ps $>0.05 ; 250 \mathrm{kD}$, Fig. 2c; $150 \mathrm{kD}$, data not shown) but significantly reduced GluA2 expression $[t(22)=2.39, p<0.05$, Fig. 2d]. Conversely, evoked AMPAR-mediated fEPSPs were significantly enhanced during AIE-withdrawal in female mice compared to air-exposed controls throughout an input-output curve of stimulation intensities [main effect of vapor: $F(1,11)=$ $6.72, p<0.05$, Supplementary Fig. 2]. These results indicate that enhanced AMPAR transmission in female mice during AIE withdrawal may result from a reduction in GluA2-containing AMPARs, compensation by other AMPA subunits, or alternative mechanisms that enhance AMPAR function. In male mice, consistent with DHPG-LTD results, mGluR5 ( $p s>0.05 ; 250 \mathrm{kD}$, Fig. 2f; $150 \mathrm{kD}$, data not shown) and GluA2 ( $p>0.05$, Fig. $2 \mathrm{~g}$ ) expression showed no difference between AIE- and air-treated groups.

AIE does not alter a1-adrenergic mediated depression in the dIBNST

Similar to group I mGluRs, a1-adrenergic receptors (ARs) are Gqlinked G-protein-coupled receptors that produce excitatory LTD. In the adult male BNST, stress and alcohol produce distinct alterations in a1-AR- and DHPG-LTD [33]. Thus, we evaluated a1AR-LTD using methoxamine (a1-AR agonist, $100 \mu \mathrm{M}, 15 \mathrm{~min}$ ) during acute AIE withdrawal. No effect of AIE treatment was observed [females: $F(1,13)=3.683, p>0.05$; males: $F(1,5)=0.094$, 
A)
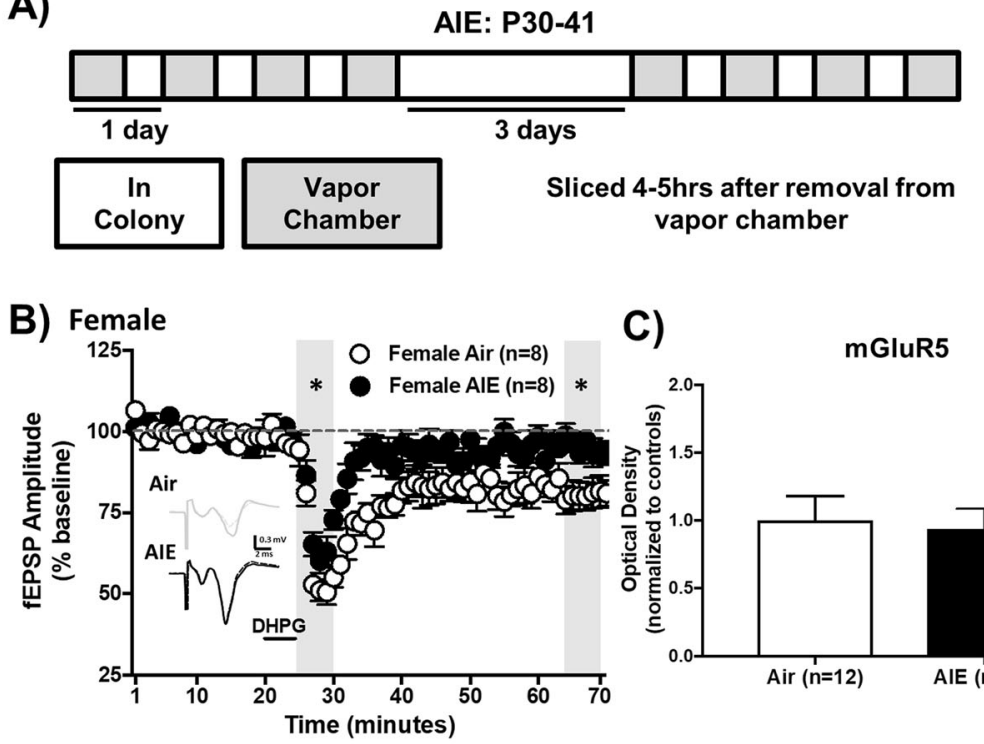

C)

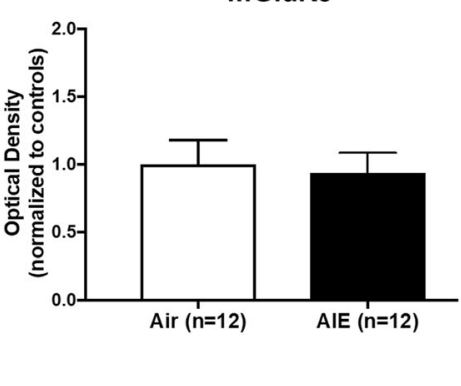

D)

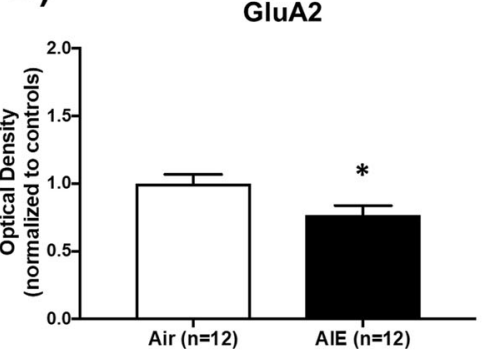

F)

mGluR5

G)

GluA2
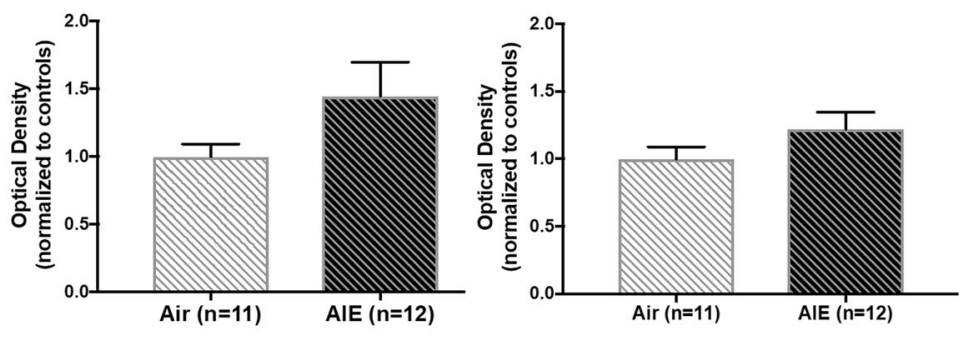

Fig. 2 DHPG-LTD, GluA2, and mGluR5 protein expression in the dBNST following acute withdrawal from AIE in female and male mice. Average time-course of field EPSP (fEPSP) amplitudes following DHPG application in females (b) and males (e), demonstrating blunted early and late component DHPG-LTD in females during AIE-withdrawal (a). Blunted DHPG-LTD was associated with significantly reduced GluA2 (d), but not mGluR5 (c) protein expression during acute AIE withdrawal compared to control levels in female mice. GluA2 (g) and mGluR5 (f) protein expression levels were not changed by acute AIE withdrawal in males. Data are shown as mean \pm SEM. Significantly different than the respective air group at ${ }^{*} p<0.05$.

$p>0.05$; Supplementary Fig. 3], indicating that a1-AR-plasticity is intact in AIE withdrawal.

AIE-attenuated mGluR-LTD does not persist into adulthood but is reestablished following stress

Persistent AIE-induced disruptions in neural plasticity could contribute to the increased susceptibility of AUD development following adolescent alcohol exposure [8, 10]. To evaluate the duration of these plasticity changes, mice were allowed to age to adulthood (P70+; Fig. 3a). At this age, no significant difference in DHPG-LTD was observed between vapor treatment groups in either sex [female early LTD: $t(13)<1, p>0.05$; female late LTD: $t(13)<1, p>0.05$; male early LTD: $t(8)=1.09, p>0.05$; male late LTD: $t(7)=2.23, p>0.05$, data not shown], revealing that AIEinduced blunting of DHPG-LTD in female mice was not preserved into adulthood.

As stress is a common trigger for relapse [10], we tested whether $1 \mathrm{~h}$ of restraint stress in adult mice with an AIE history would produce sex-specific disruptions in BNST plasticity (Fig. 3a). In stressed adult females, there was a significant effect of AIE history $[F(1,11)=11.96, p<0.01]$ and DHPG-LTD timepoint [early or late phase, $F(1,11)=30.72, p<0.001$ ] with no interaction $(p>$ 0.05, Fig. 3b). In contrast to the blunted DHPG-LTD observed in AlE-exposed females during acute withdrawal (Fig. 2b), stress during protracted withdrawal significantly enhanced DHPG-LTD compared to the stressed air group (early: Sidak $p<0.05$, late: $p=$ 0.09 ). Notably, although spontaneous EPSC amplitude is enhanced in AIE-exposed females compared to air controls following stress [21], AIE and stress did not alter evoked AMPAR-mediated fEPSPs $[F(1,5)<1, p>0.05$; Supplementary Fig. 4]. In stressed adult males, there was no significant effect of AIE history ( $p>0.05$, Fig. $3 c$ ). Thus, acute stress during adulthood selectively impaired induction and maintenance of mGluR-LTD in the dIBNST of females, but not males, with a history of AIE.

DHPG-induced LTD in the adult male BNST is mediated primarily by mGluR5 [22]. In naïve adult female mice, MTEP (mGluR5 antagonist, $10 \mu \mathrm{M}$ ) was applied prior to DHPG during fEPSPs. This antagonism significantly reduced the early LTD component and slightly potentiated the late component of LTD [one-sample $t$ test compared to $100 \%$; early: $t(3)=11.62, p<0.01$; late: $t(3)=5.64, p<0.05$; Fig. $3 \mathrm{~d}$, e]. These results show that, similar to adult males, mGluR5 is the primary regulator of DHPG-LTD in the BNST of adult females.

AIE history does not alter basal anxiety-like activity in adulthood Basal anxiety-like activity was measured in the open field of the fear chamber by calculating the \% of total distance traveled in the center field $3 \mathrm{~min}$ prior to shock exposure. AIE-exposure did not 
A) AIE: P30-41

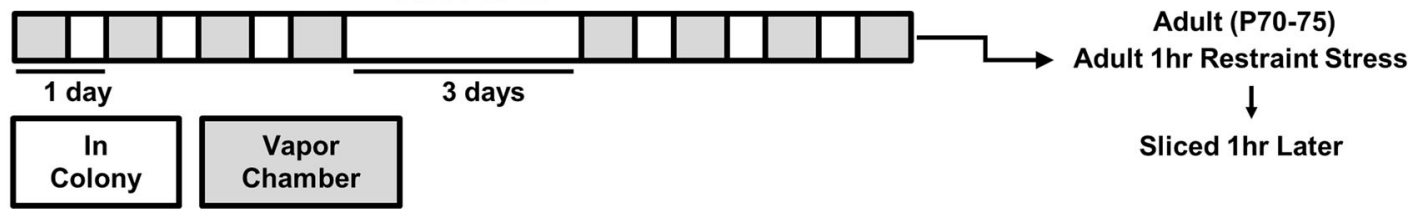

B) Female - Stress

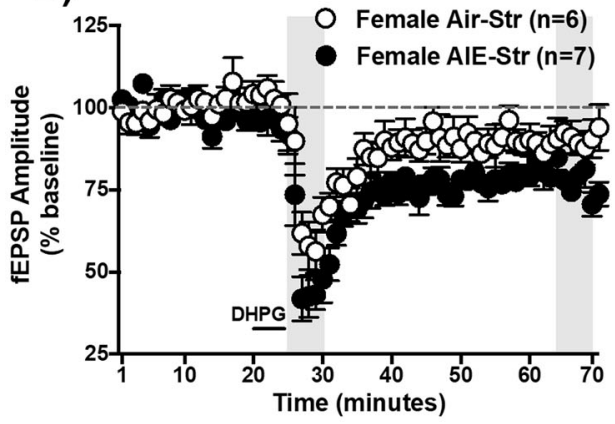

D) Naïve Adult female - No Stress

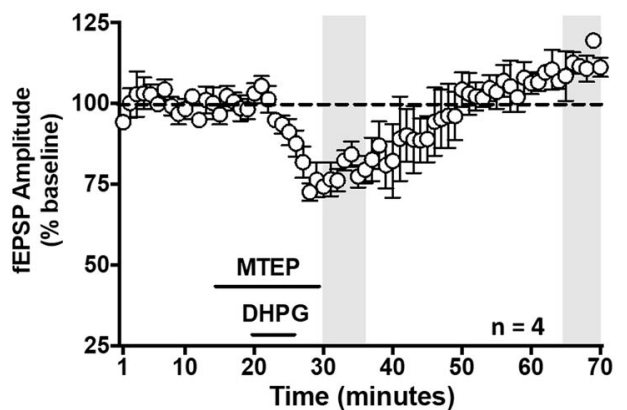

C) Male - Stress

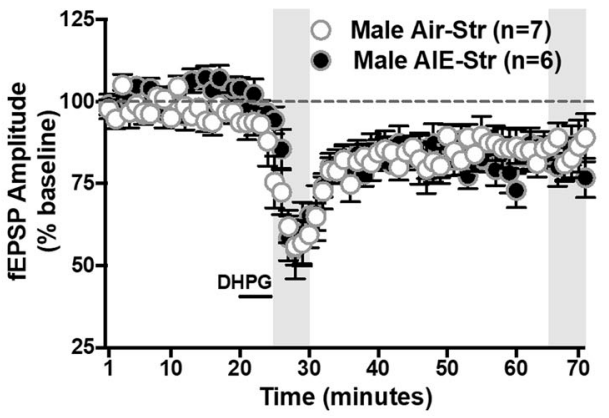

E) Naïve Adult female - No Stress

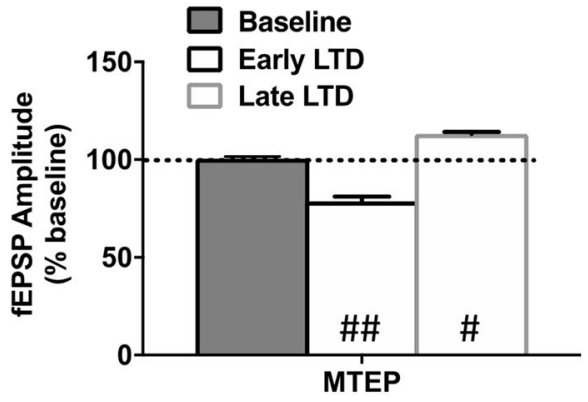

Fig. 3 Restraint stress uncovers persistent alterations in DHPG-LTD in the dIBNST of adult females during protracted withdrawal from AIE. Following adolescent vapor exposure, mice were allowed to rest until adulthood before slices containing the dIBNST were collected (a). In females, stress uncovered enhanced DHPG-LTD during the early phase (b). In males, AIE history did not alter DHPG-LTD under stress conditions (c). Average time-course of fEPSP amplitudes when the mGluR5 antagonist MTEP was applied prior to DHPG-induced LTD in naïve female mice (d). MTEP reduced the early phase component and potentiated the late phase component of LTD (e). Data are shown as mean \pm SEM. Significantly different than the respective air group at ${ }^{*} p<0.05$; significantly different than $100 \%$ at ${ }^{\#} p<0.05$, ${ }^{\# \#} p<0.01$.

significantly alter \% center distance in females $[t(11)=1.045, p>$ 0.05 ] or males $[t(13)=0.61, p>0.05$; Fig. 4ai, ii], indicating no persistent changes in basal anxiety-like activity.

AIE history and adult restraint stress sex-dependently mediate anhedonia-like behavior in the NIH task

The $\mathrm{NIH}$ task is a preclinical analog of negative affect-associated despair and anhedonia. Despair-like behavior can be decreased via reductions in mGluR5 phosphorylation in the BNST [34]. Although the NIH task has not been directly linked to mGluR5s, it can be bidirectionally mediated by stressors and pharmaceutical intervention and is dependent upon GluN2B activity in the BNST $[29,35]$. Mice were tested for $\mathrm{NIH}$ during adulthood $(\mathrm{P} 70+)$ following AIE exposure (Fig. 4a). To account for individual and intra-sex variability in consummatory latencies [5, 36], latencies on the test day were quantified as change in consummatory latency from the training day. Training latencies and amount of Ensure consumed during the test session are shown in Supplementary Figs. 5-6.

Under basal (no-stress) conditions, the novel test environment significantly increased latency to consume in air-exposed females and males [one-way $t$ tests compared to $0, p s<0.05$ ] and a trend for increased latency in AIE-exposed females and males [females: $p=0.09$; males: $p=0.07$ Fig. $4 \mathrm{bi}$, ii]. Induction of a negative affective-like state using restraint stress coupled with the novel test environment significantly increased latency to consume in all groups [one-way $t$ tests compared to $0, p s<0.01$, Fig. $4 \mathrm{bi}$, ii].

In females, a stress*vapor history ANOVA revealed a weak trend toward a main effect of alcohol $(p=0.08)$ and no significant main effect of stress $(p>0.05)$ or interaction $(p=0.11)$ on change in latency to consume on the test day (Fig. 4bi). Planned comparisons within stress status revealed no effect of AIE history within the non-stressed group (Sidak $p>0.05$ ), but a stress potentiated anhedonia-like state in females with an AIE history compared to their air-control counterparts (Sidak $p<0.05$ ).

In males, a stress*vapor history ANOVA revealed no significant main effects or interaction ( $p s>0.05$ ) on change in latency to consume on the test day (Fig. 4bii). Planned comparisons revealed no effect of AIE history within the non-stressed or stressed groups (Sidak ps >0.05). Notably, a larger proportion of AlE-exposed males did not consume on the second training day prior to stress exposure (Supplementary Table 1) and also showed a trend towards increased latency to consume on the training day (Supplementary Fig. 5b). This potentially indicates an effect of AIE on neophobia in males.

mGluR5 antagonism reliably promotes an anxiolytic phenotype in rodents $[37,38]$. Further, it is the key regulator of BNST mGluRLTD [23] and altering BNST mGluR5 phosphorylation reduces 

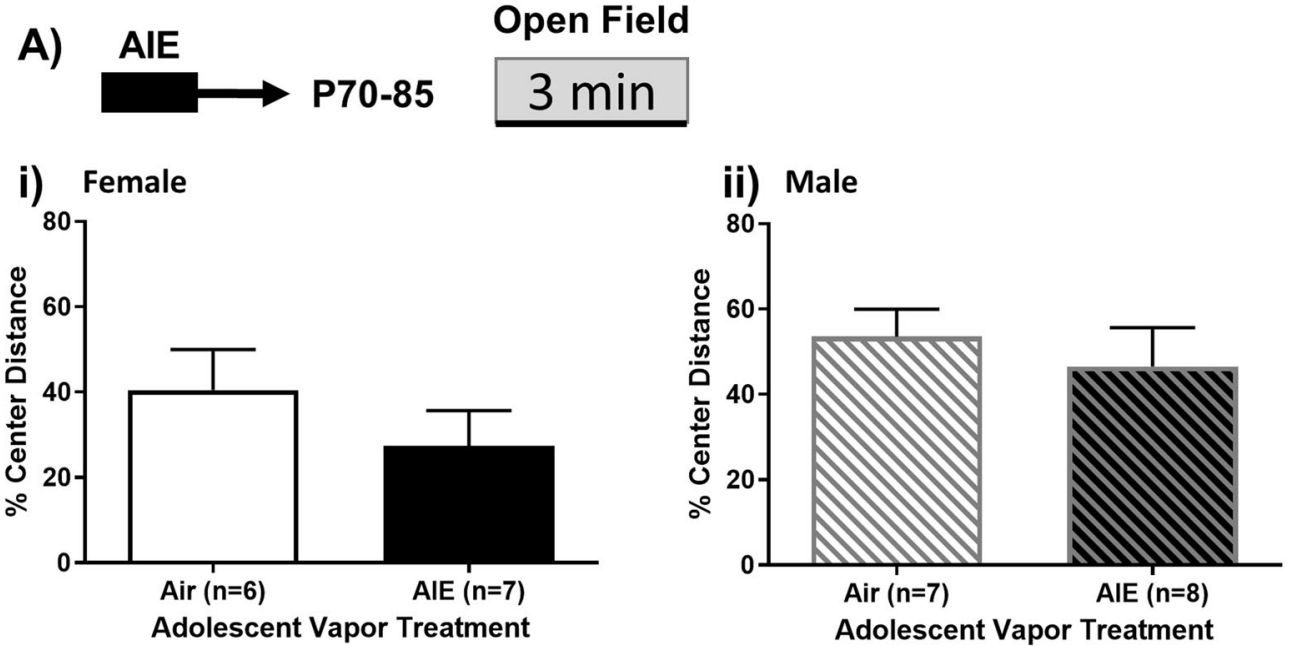

B)

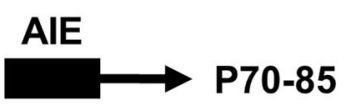

MTEP Restraint Stress

i) Female - No pretreatment

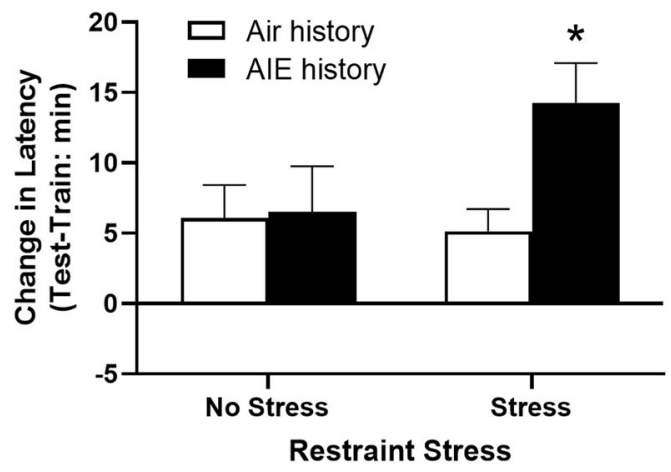

ii) Male - No pretreatment

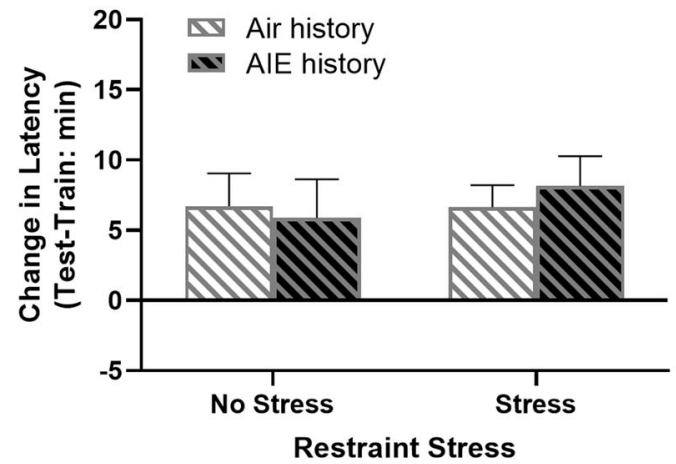

iii) Female - Drug pretreatment and stress

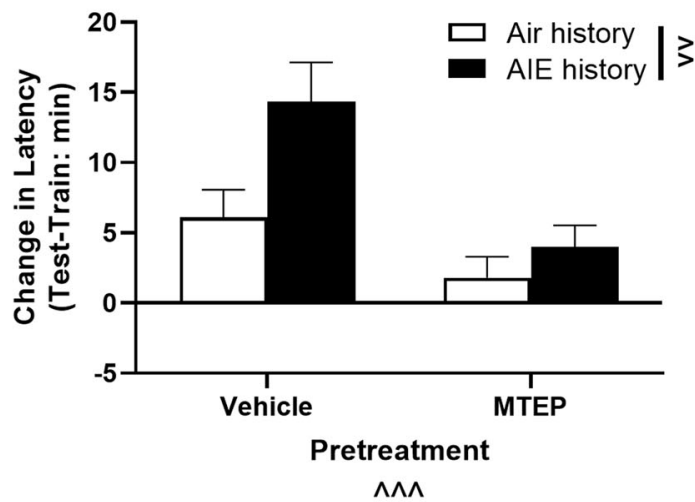

Fig. 4 Restraint stress produces persistent changes in negative-affective-like states that are not present under basal conditions. a Following adolescent vapor exposure, mice were allowed to rest until adulthood before being exposed to the open field. Protracted AIE withdrawal did not alter anxiety-like activity in the open field in females (Ai) or males (Aii). In the NIH task, mice were exposed to $1 \mathrm{~h}$ of restraint stress or kept in their home cage. AlE-exposed females (Bi), but not males (Bii), exposed to restraint stress significantly enhanced their latency to consume an appetitive reinforcer compared to their air counterparts, ns $=6-8$ in females and 7-10 in males. To observe whether mGlu5 receptors mediate this behavior in female mice, vehicle or $30 \mathrm{mg} / \mathrm{kg}$ MTEP was injected 30 min prior to restraint stress (Biii). MTEP administration blocked stress-induced enhancements in latency to consume, $\mathrm{ns}=7-11$. Data are shown as mean \pm SEM. Significant main effect at $\wedge \wedge p<0.01$ and $\wedge \wedge \wedge p<0.001$. Significantly different than the respective control at ${ }^{*} p<0.05$. 
A)

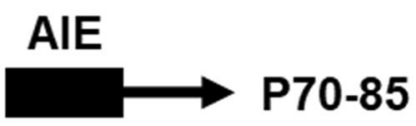

Contextual Fear

B) Female - Freezing Acquisition
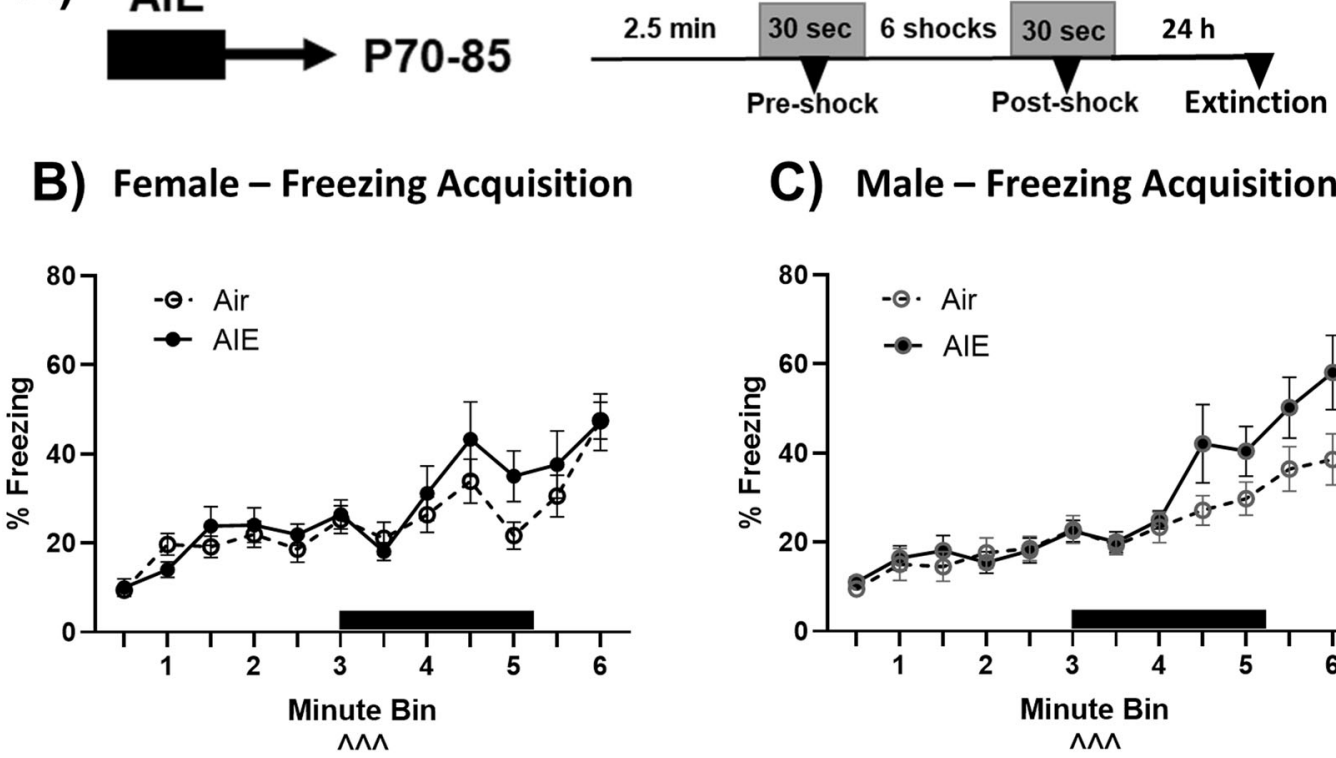

\section{D) Female - Binned Freezing}
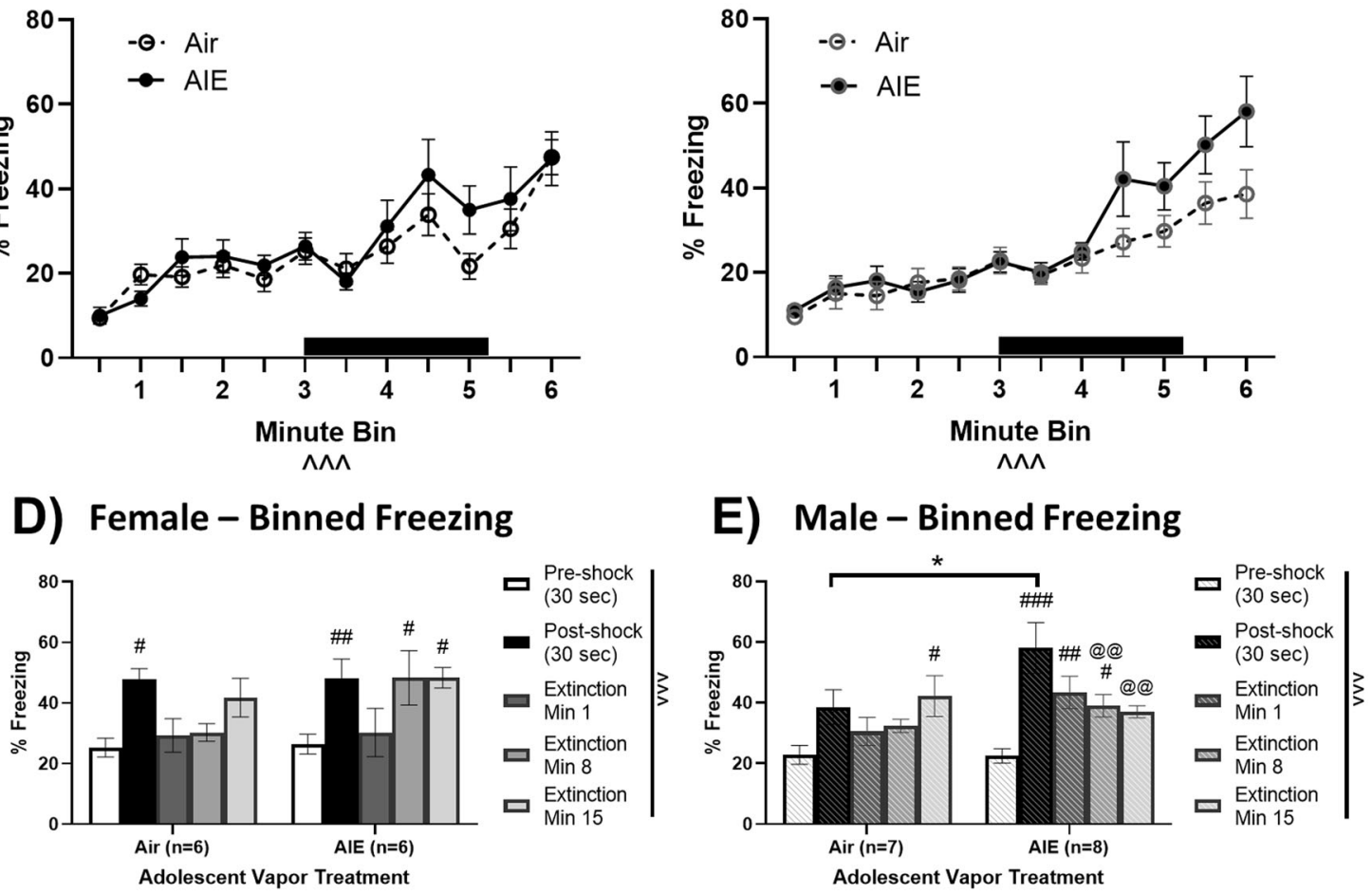

Fig. 5 AIE-exposure enhances contextual fear behavior in male mice. Following adolescent vapor exposure, mice were allowed to rest until adulthood before being exposed to low-level foot shocks (a). Acquisition of freezing activity during the training session in females (b) and males (c). The black bar represents the period of shock exposure. Binned freezing activity in females (d) and males (e) in the contextual fear conditioning task. AlE-exposed males showed significantly increased fear acquisition compared to their air counterparts that was not present in females. Data are shown as mean \pm SEM. Significant main effect at $\wedge \wedge \wedge p<0.001$. Significantly different than the respective air group at ${ }^{*} p<$ 0.05 . Significantly different than respective pre-shock freezing at ${ }^{\#} p<0.05,{ }^{\# \#} p<0.01, \# \# p<0.001$. Significantly different than respective postshock freezing at ${ }^{@} p<0.01$.

despair-like behavior [34]. Therefore, we tested the efficacy of a systemic vehicle or MTEP pretreatment $30 \mathrm{~min}$ prior to stress to blunt AIE-induced enhancement of consummatory latency in female mice (Fig. 4biii). A drug*vapor history ANOVA revealed significant main effects of drug $[F(1,33)=14.80, p<0.001]$ and vapor $[F(1,33)=7.49, p<0.01]$, but no interaction $(p>0.05)$. All drug pretreated females showed an increased consummatory latency compared to training except for air-exposed MTEP-treated mice [one sample $t$ test; air history, vehicle: $t(8)=3.11, p<0.05$; AIE history, vehicle: $t(6)=5.07, p<0.01$; air history, MTEP: $t(9)=$ $1.12, p>0.05$, AIE history, MTEP: $t(10)=2.62, p<0.05$ ].

AIE history sex-dependently mediates contextual fear conditioning BNST activity regulates contextual fear behavior in male rodents $[39,40]$. To probe for effects of AIE history on increased threat monitoring following a low-intensity foot shock, we observed freezing at pre-shock, post-shock, and subsequent contextual reexposure and extinction session (mins 1, 8, and 15, no shocks). Females and males were analyzed separately due to the use of disparate shock intensities, which were necessary to produce similar levels of freezing between groups (Fig. 5a; see Supplementary Materials). Detailed time*vapor history mixed effects analyses are presented in Supplementary Fig. 7. Briefly, shock exposure increased freezing over the training session in both sexes (Fig. 5b, c;
Supplementary Fig. 7a, c). In females, low-intensity foot shocks increased freezing behavior compared to pre-shock levels regardless of vapor history (Sidak $p s<0.05$ ), but this increased freezing behavior did not persist in a subsequent recall test (extinction min 1, Sidak $p s>0.05$, Fig. $5 \mathrm{~d}$ ). In males, low-intensity foot shocks did not significantly alter freezing activity at postshock or at extinction min 1 in air-history mice (Sidak $p s>0.05$ ), but did increase freezing at these timepoints in mice with an AIE history (Sidak $p s<0.01$; Fig. 5e). Notably, in males, the AIE-exposed group exhibited a significantly greater change in post-shock freezing compared to the air-exposed group $[t(13)=2.34$, $p<0.05]$. These results indicate elevated, persistent threat monitoring in AlE-exposed males following low-intensity foot shocks that was not present in their air counterparts or AIE-exposed females.

\section{DISCUSSION}

Females are an historically understudied population, even in investigations of disorders that disproportionately affect them [41]. Given the pervasiveness of adolescent drinking in both sexes [42], and the significance of the adolescent period for developing AUDs and comorbid disorders $[6,9]$, the need to understand sex differences in alcohol's long-term neurological and behavioral effects is critical. Acute AIE withdrawal increased anxiety-like 
activity and produced hypo-locomotion in both sexes, and our previous work demonstrates that spontaneous glutamatergic activity in the BNST is increased in both sexes at this timepoint. However, NMDAR-mediated changes were specific to male mice [21], whereas the current data demonstrated that disruptions in group I mGluR-mediated BNST LTD were specific to female mice. Although persistent changes in group I mGluR-LTD were not present under basal conditions in adulthood, they were unmasked by stress. Stressed females with an AIE history also exhibited increased latency to consume an appetitive reinforcer, which was not present under basal conditions. Conversely, adult males with an AIE history displayed increased freezing in a low-intensity contextual fear paradigm that was not observed in females. These results indicate that AIE produces persistent sex-dependent disruptions in behavior and BNST plasticity that are unmasked by stress.

\section{Sex differences in group I mGluR-LTD}

Acute AIE withdrawal selectively impaired induction and maintenance of group I mGluR-LTD in the dIBNST of female, but not male, mice. These effects could be explained by one of the following: (1) mGluR-LTD-generating mechanisms being blunted by AIE (e.g., reduced mGluR1/5 or Erk) or (2) the occurrence of LTD in vivo, prior to in vitro induction via DHPG, and therefore further depression could not be elicited. Because we observed a femalespecific reduction in GluA2, but not mGluR5, this points toward the latter. At this same time point, however, AMPAR-mediated fEPSPs were enhanced compared to air controls, which would be counter to this hypothesis. Therefore, the mechanisms regulating this AIE-induced change in LTD remain unknown. During the adult timepoint, AIE's effects on group I mGluR-LTD were absent under non-stressed conditions but enhanced in females after stress. It is likely that AIE history and stress act through a shared mechanism to enhance second messenger signaling cascades, converging to augment group I mGluR-LTD in females. The nature of this sexspecific effect remains unclear, but perhaps divergence in downstream second messenger cascades are responsible. As previous studies implicating a role for mGluR-LTD in addiction mainly focus on adult male rodents [22-24, 27], our findings provide novel insight that suggest factors determining the induction and expression of alcohol-related mGluR plasticity likely diverge across both age and sex.

Sex differences in stress-induced negative affective-like behavior Negative affect-characterized by stress, anxiety, anhedonia, and hypervigilant states -is associated with alcohol withdrawal and craving and BNST dysfunction [4, 17]. In the current work, males and females displayed anxiety-like activity on the EPM during acute AIE withdrawal. This anxiety-like phenotype from early alcohol use may prime individuals to insults that occur later in life. Indeed, while AIE history had no effect on anxiety- or anhedonialike behavior under basal conditions in adults, restraint stress or low-intensity foot shock [30] uncovered persistent AIE-dependent changes.

The results of the $\mathrm{NIH}$ task are consistent with the literature in that no differences were observed across sex in control mice [43-47]. Although aversive conditions on the test day increased latency to consume in all groups, this effect did not reach significance in non-stressed AIE-exposed males or females. Restraint stress induced a significantly greater consumption latency in AIE-exposed females compared to their air-exposed counterparts that was not present in males. This may indicate that acute restraint stress is a relatively low-level stressor that AIEexposed females are sensitive to. Work using the similar noveltysuppressed feeding task reports a significant increase in latency to consume in adult mice undergoing withdrawal from voluntary ethanol exposure. Interestingly, the increased latency is present during a relatively short period of abstinence in males, but requires longer periods of abstinence in females [48-52]. As AIEexposure did not increase consumption latency in males during adulthood, this suggests that anhedonia-like behavior may be more immediate and transient in males, but that it kindles in females. It should be noted that a larger proportion of AIE males were excluded from the restraint stress $\mathrm{NIH}$ analyses due to failure to consume during the training phase, and those who did consume during training trended towards having a higher latency to first consumption. As these metrics were collected prior to stress, they may indicate that a subpopulation of AlE-treated males experienced a unique negative affective-like state that made them particularly sensitive to novel stimuli. Further studies are needed to fully explore sex differences and individual variation in the phenotypic expression of negative affective-like behavior.

Electrophysiological data revealed altered DHPG-LTD in the dIBNST of AIE-treated females, but not males, after adult stress and this LTD was mediated by mGluR5. We further found that MTEP pretreatment in females blunted the effects of stress in the $\mathrm{NIH}$ test. Several studies have similarly linked mGluR5 signaling to stress and alcohol effects during adolescence. Adolescent social isolation (a chronic stressor) produces anxiety-like behavior associated with increased mGluR5-mediated excitability in the basolateral amygdala [53]. mGluR5 modulation has also been shown to reverse aberrant AIE-induced ethanol self-administration [54] and binge drinking during adolescence-the effect of which is more pronounced in females [25]. A recent study which blocked mGluR5 phosphorylation by Erk reported excessive drinking and reduced anxiety- and despair-like activity in mice, potentially through actions in the BNST [34]. mGluR5 has long been proposed as a pharmacological target to treat alcohol use and comorbid disorders $[26,55]$. In humans, genetic variants in the mGluR-eEF2AMPAR pathway, which includes mGluR5, have been associated with fewer number of drinking days in the past month [56]. While a number of mGluR5 negative allosteric modulators have been developed for clinical use [57], their potential to alleviate symptoms associated with alcohol use and comorbid disorders has not yet been investigated.

\section{Sex differences in contextual fear}

Contextual fear conditioning is an animal model of threat monitoring elicited by unpredictable, aversive stimuli [16]. The BNST's role in unpredictable shock response is conserved across male rodents [39,40] and humans of both sexes [58]. We found that AIE-exposed males showed enhanced contextual fear acquisition to low-intensity foot shocks relative to their control counterparts and that this enhanced freezing behavior persisted into the extinction trial. Interestingly, elevated freezing behavior was not present in AIE-females. Our previous work demonstrated that AIE sex-dependently disrupts GluN2B-mediated signaling in the BNST of male mice [21]. Contextual fear conditioning stimulates Fyn kinase, which increases GluN2B phosphorylation in the hippocampus and mediates contextual fear extinction [59-61]. It is therefore plausible that GluN2B receptors of the BNST mediate enhanced fear activity in males. Ethanol's reported effects on contextual fear memory in adolescent and adult rodents is mixed depending on the ethanol/withdrawal paradigm used [62-65]. Broadwater and Spear [62] utilized a protocol most similar to the current study, yet their results yielded no differences in adult fear conditioning following adolescent ethanol in male rats. Methodological disparities in ethanol exposure (intragastric vs. vapor), context conditioning levels (different levels of freezing), and rodent type (rat vs. mouse) could contribute to the divergent effects.

\section{CONCLUSION}

Our findings demonstrate distinct sex-dependent effects of AIE during acute and protracted withdrawal from ethanol. These 
effects are dependent on stress during protracted withdrawal, further supporting the complicated relationship between alcohol use, negative affect, stress, and relapse. The mechanisms regulating AIE-induced long-term changes in BNST plasticity are divergent in male (GluN2B-mediated) [21] and female (mGluR1/5) mice, which may contribute to the observed sex differences in behavioral phenotypes. The current studies demonstrate that stress unveils a negative affective state that can be pharmaceutically rescued by mGluR5 antagonism in females, lending support to the use of pharmaceutical intervention with mGluR5 in women suffering from AUDs and comorbid disorders. Further, these results implicate distinct mechanisms in the sexually dimorphic BNST that regulate acute and protracted AIE withdrawal and its associated behavioral phenotypes.

\section{FUNDING AND DISCLOSURES}

This work was supported by the National Institute on Alcohol Abuse and Alcoholism (K99/R00 AA022651 and T32 AA007577). The authors declare that the research was conducted in the absence of any commercial or financial relationships that could be construed as a potential conflict of interest.

\section{AUTHOR CONTRIBUTIONS}

TW and CK were responsible for the study concept and design. TW, KC, EH, CK, and NS conducted the electrophysiological recordings. MM and ML performed the vapor chamber, acute restraint stress, western blotting, and slicing procedures. $\mathrm{CK}, \mathrm{EH}$, and TH conducted the behavioral experiments. All authors assisted with data analysis. TW, $\mathrm{CK}$, and $\mathrm{KC}$ were responsible for the interpretation of findings, and $\mathrm{KC}$ and $\mathrm{CK}$ drafted and revised the paper. TW provided critical revision of the paper for important intellectual content. All authors critically reviewed content and approved the final version for publication.

\section{ADDITIONAL INFORMATION}

Supplementary Information accompanies this paper at (https://doi.org/10.1038/ s41386-020-0670-7).

Publisher's note Springer Nature remains neutral with regard to jurisdictional claims in published maps and institutional affiliations.

\section{REFERENCES}

1. Thomasson HR. Gender Differences in Alcohol Metabolism. Recent Development in Alcoholism. p. 163-79. Boston, MA, USA: Springer; 2002.

2. Devaud LL, Alele P, Ritu C. Sex differences in the central nervous system actions of ethanol. Crit Rev Neurobiol. 2003;15:41-59.

3. Becker JB, McClellan ML, Reed BG. Sex differences, gender and addiction. J Neurosci Res. 2017;95:136.

4. Peltier MR, Verplaetse TL, Mineur YS, Petrakis IL, Cosgrove KP, Picciotto MR, et al. Sex differences in stress-related alcohol use. Neurobiol Stress. 2019;10:100149.

5. Rubinow DR, Schmidt PJ. Sex differences and the neurobiology of affective disorders. Neuropsychopharmacology. 2019;44:111-28.

6. Craske MG, Stein MB, Eley TC, Milad MR, Holmes A, Rapee RM, et al. Anxiety disorders. Nat Rev Dis Prim. 2017;3:17024.

7. McHugh RK, Votaw VR, Sugarman DE, Greenfield SF. Sex and gender differences in substance use disorders. Clin Psychol Rev. 2018;66:12-23.

8. Grant BF, Dawson DA. Age at onset of alcohol use and its association with DSM-IV alcohol abuse and dependence: results from the National Longitudinal Alcohol Epidemiologic Survey. J Subst Abus. 1997;9:103-10.

9. Nixon K, McClain JA. Adolescence as a critical window for developing an alcohol use disorder: current findings in neuroscience. Curr Opin Psychiatry. 2010;23:227-32.

10. Becker HC. Influence of stress associated with chronic alcohol exposure on drinking. Neuropharmacology. 2017;122:115-26.

11. Crews FT, Robinson DL, Chandler LJ, Ehlers CL, Mulholland PJ, Pandey SC, et al. Mechanisms of persistent neurobiological changes following adolescent alcohol exposure: NADIA consortium findings. Alcohol Clin Exp Res. 2019;43:1806-22.

12. Brown TA, Barlow DH. A proposal for a dimensional classification system based on the shared features of the DSM-IV anxiety and mood disorders: implications for assessment and treatment. Psychol Assess. 2009;21:256-71.
13. Kirlic N, Aupperle RL, Rhudy JL, Misaki M, Kuplicki R, Sutton A, et al. Latent variable analysis of negative affect and its contributions to neural responses during shock anticipation. Neuropsychopharmacology. 2019;44:695-702.

14. Wright JS, Panksepp J. Toward affective circuit-based preclinical models of depression: sensitizing dorsal PAG arousal leads to sustained suppression of positive affect in rats. Neurosci Biobehav Rev. 2011;35:1902-15.

15. Goodwill HL, Manzano-Nieves G, Gallo M, Lee H-I, Oyerinde E, Serre T, et al. Early life stress leads to sex differences in development of depressive-like outcomes in a mouse model. Neuropsychopharmacology. 2019;44:711-20.

16. Ganella DE, Kim JHYU. Developmental rodent models of fear and anxiety: from neurobiology to pharmacology. Br J Pharm. 2014;171:4556-74.

17. Lebow MA, Chen A. Overshadowed by the amygdala: the bed nucleus of the stria terminalis emerges as key to psychiatric disorders. Mol Psychiatry 2016;21:450-63.

18. Koob GF, Volkow ND. Neurocircuitry of addiction. Neuropsychopharmacology. 2010;35:217-38.

19. Wills TA, Kash TL, Winder DG. Developmental changes in the acute ethanol sensitivity of glutamatergic and GABAergic transmission in the BNST. Alcohol 2013;47:531-7.

20. Wills TA, Klug JR, Silberman Y, Baucum AJ, Weitlauf C, Colbran RJ, et al. GluN2B subunit deletion reveals key role in acute and chronic ethanol sensitivity of glutamate synapses in bed nucleus of the stria terminalis. Proc Natl Acad Sci USA. 2012;109:E278-E287

21. Carzoli KL, Sharfman NM, Lerner MR, Miller MC, Holmgren EB, Wills TA. Regulation of NMDA receptor plasticity in the BNST following adolescent alcohol exposure. Front Cell Neurosci. 2019;13:224.

22. Grueter BA, Gosnell HB, Olsen CM, Schramm-Sapyta NL, Nekrasova T, Landreth $\mathrm{GE}$, et al. Extracellular-signal regulated kinase 1-dependent metabotropic glutamate receptor 5 -induced long-term depression in the bed nucleus of the stria terminalis is disrupted by cocaine administration. J Neurosci. 2006:26:3210-9.

23. Grueter BA, McElligott ZA, Robison AJ, Mathews GC, Winder DG. In vivo metabotropic glutamate receptor 5 (mGluR5) antagonism prevents cocaine-induced disruption of postsynaptically maintained mGluR5-dependent long-term depression. J Neurosci. 2008;28:9261-70.

24. Lominac KD, Kapasova Z, Hannun RA, Patterson C, Middaugh LD, Szumlinski KK. Behavioral and neurochemical interactions between Group 1 mGluR antagonists and ethanol: potential insight into their anti-addictive properties. Drug Alcohol Depend. 2006;85:142-56.

25. Cozzoli DK, Strong-Kaufman MN, Tanchuck MA, Hashimoto JG, Wiren KM, Finn DA. The effect of mGluR5 antagonism during binge drinking on subsequent ethanol intake in C57BL/6J mice: Sex- and age-induced differences. Alcohol Clin Exp Res. 2014;38:730-8.

26. Kasten CR, Holmgren EB, Wills TA. Metabotropic glutamate receptor subtype 5 in alcohol-induced negative affect. Brain Sci. 2019;9:183.

27. Wills TA, Baucum AJ, Holleran KM, Chen Y, Pasek JG, Delpire E, et al. Chronic intermittent alcohol disrupts the GluN2B-associated proteome and specifically regulates group I mGlu receptor-dependent long-term depression. Addict Biol. 2017;22:275-90.

28. Kasten CR, Zhang Y, Boehm SL. Acute cannabinoids produce robust anxiety-like and locomotor effects in mice, but long-term consequences are age- and sexdependent. Front Behav Neurosci. 2019;13:1-18.

29. Dulawa SC, Hen R. Recent advances in animal models of chronic antidepressant effects: the novelty-induced hypophagia test. Neurosci Biobehav Rev. 2005;29:771-83.

30. Heinrichs SC, Koob GF. Application of experimental stressors in laboratory rodents. Curr Protoc Neurosci. 2006; Chapter 8: Unit 8.4

31. Heilig M, Egli M, Crabbe JC, Becker HC. Acute withdrawal, protracted abstinence and negative affect in alcoholism: are they linked? Addict Biol. 2010;15:169-84.

32. Vranjkovic O, Pina M, Kash TL, Winder DG. The bed nucleus of the stria terminalis in drug-associated behavior and affect: a circuit-based perspective. Neuropharmacology 2017;122:100-6.

33. McElligott ZA, Klug JR, Nobis WP, Patel S, Grueter BA, Kash TL, et al. Distinct forms of Gq-receptor-dependent plasticity of excitatory transmission in the BNST are differentially affected by stress. Proc Natl Acad Sci USA. 2010;107:2271-6.

34. Campbell RR, Domingo RD, Williams AR, Wroten MG, McGregor HA, Waltermire RS, et al. Increased alcohol-drinking induced by manipulations of mglu5 phosphorylation within the bed nucleus of the stria terminalis. J Neurosci. 2019;39:2745-61.

35. Louderback KM, Wills TS, Muglia LJ, Winder DG. Knockdown of BNST GluN2Bcontaining NMDA receptors mimics the actions of ketamine on novelty-induced hypophagia. Transl Psychiatry. 2013;3:e331.

36. Shansky RM. Sex differences in PTSD resilience and susceptibility: challenges for animal models of fear learning. Neurobiol Stress 2015;1:60-5. 
37. Riaza Bermudo-Soriano C, Perez-Rodriguez MM, Vaquero-Lorenzo C, Baca-Garcia E. New perspectives in glutamate and anxiety. Pharm Biochem Behav. 2012;100:752-74.

38. Lee KM, Coelho MA, Class MA, Szumlinski KK. mGlu5-dependent modulation of anxiety during early withdrawal from binge-drinking in adult and adolescent male mice. Drug Alcohol Depend. 2018;184:1-11.

39. Sullivan GM, Apergis J, Bush DEA, Johnson LR, Hou M, Ledoux JE. Lesions in the bed nucleus of the stria terminalis disrupt corticosterone and freezing responses elicited by a contextual but not by a specific cue-conditioned fear stimulus. Neuroscience. 2004;128:7-14.

40. Zimmerman JM, Maren S. The bed nucleus of the stria terminalis is required for the expression of contextual but not auditory freezing in rats with basolateral amygdala lesions. Neurobiol Learn Mem. 2011;95:199-205.

41. Zucker I, Beery AK. Males still dominate animal studies. Nature. 2010;465:690.

42. Johnson JK, Johnson RM, Hodgkin D, Jones AA, Matteucci AM, Harris SK. Heterogeneity of state medical marijuana laws and adolescent recent use of alcohol and marijuana: analysis of 45 states, 1991-2011. Subst Abus. 2018;39:247-54.

43. Savarese A, Lasek AW. Regulation of anxiety-like behavior and Crhr1 expression in the basolateral amygdala by LMO3. Psychoneuroendocrinology 2018;92:13-20.

44. Fisher ML, LeMalefant RM, Zhou L, Huang G, Turner JR. Distinct roles of CREB within the ventral and dorsal hippocampus in mediating nicotine withdrawal phenotypes. Neuropsychopharmacology. 2017;42:1599-609.

45. Yamada D, Wada E, Amano T, Wada K, Sekiguchi M. Lack of neurotensin type 1 receptor facilitates contextual fear memory depending on the memory strength. Pharm Biochem Behav. 2010;96:363-9.

46. Kiselycznyk C, Zhang X, Huganir RL, Holmes A, Svenningsson P. Reduced phosphorylation of GluA1 subunits relates to anxiety-like behaviours in mice. Int J Neuropsychopharmacol. 2013;16:919-24.

47. Sun Y, Zupan B, Raaka BM, Toth M, Gershengorn MC. TRH-receptor-type-2-deficient mice are euthyroid and exhibit increased depression and reduced anxiety phenotypes. Neuropsychopharmacology. 2009;34:1601-8.

48. Pang TY, Renoir T, Du X, Lawrence AJ, Hannan AJ. Depression-related behaviours displayed by female C57BL/6J mice during abstinence from chronic ethanol consumption are rescued by wheel-running. Eur J Neurosci. 2013;37:1803-10.

49. Holleran KM, Wilson HH, Fetterly TL, Bluett RJ, Centanni SW, Gilfarb RA, et al. Ketamine and MAG lipase inhibitor-dependent reversal of evolving depressivelike behavior during forced abstinence from alcohol drinking. Neuropsychopharmacology. 2016;41:2062-71.

50. Jury NJ, DiBerto JF, Kash TL, Holmes A. Sex differences in the behavioral sequelae of chronic ethanol exposure. Alcohol. 2017;58:53-60.

51. Sidhu $H$, Kreifeldt $M$, Contet $C$. Affective disturbances during withdrawal from chronic intermittent ethanol inhalation in $\mathrm{C} 57 \mathrm{BL} / 6 \mathrm{~J}$ and $\mathrm{DBA} / 2 \mathrm{~J}$ male mice. Alcohol Clin Exp Res. 2018;42:1281-90
52. Vranjkovic O, Winkler G, Winder DG. Ketamine administration during a critical period after forced ethanol abstinence inhibits the development of time-dependent affective disturbances. Neuropsychopharmacology. 2018; 43:1915-23.

53. Lin $\mathrm{S}$, Li $X$, Chen $\mathrm{Y}-\mathrm{H}$, Gao $\mathrm{F}$, Chen $\mathrm{H}, \mathrm{Hu} \mathrm{N}-\mathrm{Y}$, et al. Social isolation during adolescence induces anxiety behaviors and enhances firing activity in BLA pyramidal neurons via mGluR5 upregulation. Mol Neurobiol. 2018;55:5310-20.

54. Gass JT, Glen WB, McGonigal JT, Trantham-Davidson H, Lopez MF, Randall PK et al. Adolescent alcohol exposure reduces behavioral flexibility, promotes disinhibition, and increases resistance to extinction of ethanol self-administration in adulthood. Neuropsychopharmacology. 2014;39:2570-83.

55. Olive MF. Metabotropic glutamate receptor ligands as potential therapeutics for addiction. Curr Drug Abus Rev. 2009;2:83-98.

56. Meyers JL, Salling MC, Almli LM, Ratanatharathorn A, Uddin M, Galea S, et al. Frequency of alcohol consumption in humans: the role of metabotropic glutamate receptors and downstream signaling pathways. Transl Psychiatry. 2015;5: e586-e586.

57. Zerbib F, Bruley des Varannes S, Roman S, Tutuian R, Galmiche J-P, Mion F, et al Randomised clinical trial: effects of monotherapy with ADX10059, a mGluR5 inhibitor, on symptoms and reflux events in patients with gastro-oesophageal reflux disease. Aliment Pharm Ther. 2011;33:911-21.

58. Somerville LH, Whalen PJ, Kelley WM. Human bed nucleus of the stria terminalis indexes hypervigilant threat monitoring. Biol Psychiatry. 2010;68:416-24.

59. Isosaka T, Kida S, Kohno T, Hattori K, Yuasa S. Hippocampal Fyn activity regulates extinction of contextual fear. Neuroreport 2009;20:1461-5.

60. Isosaka T, Hattori K, Kida S, Kohno T, Nakazawa T, Yamamoto T, et al. Activation of Fyn tyrosine kinase in the mouse dorsal hippocampus is essential for contextual fear conditioning. Eur J Neurosci. 2008;28:973-81.

61. Kojima N, Sakamoto T, Endo S, Niki H. Impairment of conditioned freezing to tone, but not to context, in Fyn-transgenic mice: relationship to NMDA receptor subunit 2B function. Eur J Neurosci. 2005;21:1359-69.

62. Broadwater M, Spear LP. Age differences in fear retention and extinction in male Sprague-Dawley rats: effects of ethanol challenge during conditioning. Behav Brain Res. 2013;252:377-87.

63. Johansson EM, García-Gutiérrez MS, Moscoso-Castro M, Manzanares J, Valverde O. Reduced contextual discrimination following alcohol consumption or MDMA administration in mice. PLoS ONE 2015;10:e0142978.

64. Ortiz V, Giachero M, Espejo PJ, Molina VA, Martijena ID. The effect of midazolam and propranolol on fear memory reconsolidation in ethanol-withdrawn rats: influence of D-cycloserine. Int J Neuropsychopharmacol. 2015;18: pyu082-pyu082.

65. Sircar R. Estrogen modulates ethanol-induced memory deficit in postpubertal adolescent rats. Alcohol Clin Exp Res. 2019;43:61-8. 